\title{
Shear Wave Dispersion Imaging for Measuring Carotid Elasticity and Viscosity
}

\author{
Zhaojun Li, MD, PhD ${ }^{a, b}$, Cuiqin Shen, MS ${ }^{b}$, Qingqing Chen, MB ${ }^{b}$, Lei Sha, MB ${ }^{b}$, Xianghong Luo, MD, PhD ${ }^{c}$, \\ Lianfang $\mathrm{Du}, \mathrm{MD}, \mathrm{PhD}{ }^{a, *}$ \\ ${ }^{a}$ Department of Ultrasound, Shanghai General Hospital, Shanghai Jiaotong University School of Medicine, Shanghai, China; \\ ${ }^{b}$ Department of Ultrasound, Shanghai General Hospital Jiading Branch, Shanghai, China; ${ }^{c}$ Department of Echocardiography, Shanghai \\ General Hospital, Shanghai Jiaotong University School of Medicine, Shanghai, China.
}

Received November 27, 2020; revision received January 30, 2021; accepted February 8, 2021

Objective: To assess the viscoelasticity of the carotid artery using shear wave dispersion Imaging and to determine the relevant factors.

Methods: Ninety volunteers were recruited and divided into elder group and young group. Shear wave dispersion (SWD) imaging was applied to measure carotid viscoelasticity. The other arterial parameters, including arterial pulse wave velocity (PWV), inner diameter (CD), intima-medium thickness (CIMT), peak systolic velocity (PSV), mean flow velocity (MFV), and velocity time integral (VTI) of the common carotid artery (CCA) were also recorded by using ultrasound measurement.

Results: Measurements showed the SWD were lower, while CD, CIMT and PWV were higher in the elder group than those of young group. However, SWD was negatively correlated with VTI, and PSV respectively $(r=-0.492$ and -0.533 , all $P<0.05$ ). SWD was also positively correlated with MFV $(r=0.354, P<0.05)$. In elder group, SWD was negatively correlated with PSV and PWV, respectively $(r=-0.372$ and -0.422 , all $P<0.05)$ while in young group, SWD positively correlated with CD, CIMT and MFV $(r=0.531,0.462$ and 0.479 respectively, and all $P<0.05)$.

Conclusion: SWD imaging shown relationship with ultrasound parameters of carotid artery and has a potential for assessment of arterial viscoelasticity.

Key words: Shear wave dispersion; Viscoelasticity; Carotid artery; Ultrasound

Advanced Ultrasound in Diagnosis and Therapy 2022; 01: 014-021

DOI: $10.37015 / A U D T .2021 .200071$

$\mathrm{M}$ echanical properties of arteries play an important role in cardiovascular function and relate to a pathological process that alters the arterial structure. In addition, stiffness is one of arterial properties and an independent predictor for many cardiovascular diseases, such as hypertension, diabetes, and stroke.

Therefore, arterial stiffness is a sign of arterial mechanical properties and may provide key information to guide therapy [1].

Artery is a viscoelastic material [2]. Though the viscosity of arterial wall plays a crucial role in storing mechanical energy generated by the heart during systole and to restoring it during diastole to optimize the heartvessel coupling, it is always neglected because of the theoretical and methodological difficulties for measuring the viscosity of artery. Hence, most existing techniques assume that the arterial wall is only of elasticity, such as pulse wave velocity (PWV). Magnetic resonance elastography has the potential to solve quantitatively both tissue elasticity and viscosity [3]. However, it has the disadvantage of lower spatial and temporal

\footnotetext{
* Corresponding authors: Department of Ultrasound, Shanghai General Hospital, Shanghai Jiaotong University School of Medicine, 100 Haining Road, Hongkou District, Shanghai 200080, China.

e-mail:dulf99@163.com unrestricted use, distribution and reproduction in any medium provided that the original work is properly attributed.
} 
resolution compared with ultrasound imaging. Shear wave elastography (SWE) has been integrated into the conventional ultrasound device and can be used to estimate arterial properties. In vitro, Maksuti et al. [4] determined the influence of confined geometry in shear modulus estimation via SWE and measured the accuracy of SWE for assessment of arterial stiffness. Couade et al. [5] exhibited the dynamic behavior of arterial wall biomechanical properties during the cardiac cycle and Maksuti et al. [6] assessed how changes in wall thickness and inner diameter influence dispersion curves of guided waves in arterial phantoms. However, in vivo, this technique has been used to measure the shear elasticity and viscosity in skeletal muscle, liver, kidney, and prostate, but the challenge remains in the artery. The purpose of this study was to illustrate the feasibility of using shear wave elastography (SWE) and dispersion (SWD) to measure arterial viscoelastic properties in different age groups. It may help in understanding the viscoelastic properties of artery, but also potentially further assessing arterial properties in the different disease processes, such as atherosclerosis, Takayasu's arteritis and aneurysm.

\section{Materials and Methods}

This was a cross-sectional study. Clinical investigations were performed according to the Declaration of Helsinki. The study protocol was approved by the ethics committee of Shanghai General Hospital (2017KY009) and registered with the official website of China Clinical Trial Registration Center (ChiCTR1800016590). The inform consents were signed by all subjects.

\section{Patient selection}

From July 2019 to June 2020, we prospectively enrolled 90 volunteers (46 men and 44 women, aged 2080 years), and they were divided into elder group $(\geqslant 50$ years old) and young group ( $<50$ years old) according to the median age. All recruited volunteers had no history of cerebrovascular events, cardiovascular events, peripheral vascular diseases, active bleeding, or malignant tumor. The enrolled subjects were required to suspend their antihypertensive medications, drinking, smoking and coffee 24 hours before the study. Then they all underwent carotid ultrasound to obtain the shear wave viscoelastic indicators and the other measurements, including arterial pulse wave velocity (PWV), inner diameter (CD) and intima-medium thickness (CIMT).

An electronic questionnaire was used to document the demographic characteristics, living habits, medical history, medication, and vascular risk factors. Diabetes mellitus was determined based on the Standards of Medical Care in Diabetes-2017 (random blood glucose $\geqslant 11.1 \mathrm{mmol} / \mathrm{L}$ or fasting blood glucose $\geqslant 7.0 \mathrm{mmol} /$ $\mathrm{L}$ ), and the diagnosis of hypertension was according to 2013 Hypertension Clinical Practice Guidelines. Blood samples were collected in the early morning following $12 \mathrm{~h}$ fasting for the test of fasting plasma glucose (FPG), triglyceride (TG), total cholesterol (TC), high-density lipoprotein cholesterol (HDL-C), low-density lipoprotein cholesterol (LDL-C), peripheral blood leucocytes, lymphocytes and neutrophils. Measured the systolic blood pressure (SBP) and diastolic blood pressure (DBP) of right brachial artery for 3 times in a calm state, then taking the mean values.

\section{SWD examination}

Common carotid artery (CCA) images were obtained using Mylab Twice ultrasound system (Esaote, Firenze, Italy) equipped with a LA523 linear transducer (4$13 \mathrm{MHz}$ ) and the software for quantitative assessment of intima-media thickness (QIMT) and arterial stiffness (QAS). In longitudinal view, the anterior and posterior carotid walls were displayed $1.0 \mathrm{~cm}$ proximal to the bulb. Pressing the "Tools" button and starting the QIMT and QAS measurements, the radiofrequency signal tracked the motion of the vascular wall for at least six cardiac cycles and calculated automatically the mean values, including CIMT, CD and PWV. In addition to these hemodynamic parameters, the peak systolic velocity (PSV), end diastolic velocity (EDV), mean flow velocity (MFV), velocity time integral (VTI), artery S/D ratio, resistance index (RI) and pulsatility index (PI) of the CCA were also recorded by using vascular ultrasound measurement. The RI, PI, and S/D ratio were calculated using the following formulas: RI $=(\mathrm{PSV}-\mathrm{EDV}) / \mathrm{PSV}, \mathrm{PI}$ $=(\mathrm{PSV}-\mathrm{EDV}) / \mathrm{MFV}$, and $\mathrm{S} / \mathrm{D}=\mathrm{PSV} / \mathrm{EDV}$.

Based on the previous study, the shear wave viscoelasticity of the CCA was assessed [7]. SWD was performed by an Aplio 900 ultrasound system (Canon Medical Systems Corporation, Otawara, Japan) with PVI-475BX curved abdominal transducer (frequency range: $1-8 \mathrm{MHz}$ and mid frequency: $5.0 \mathrm{MHz}$ ).

Electrocardiography was recorded by synchronization. Arterial viscoelasticity could be evaluated after starting "TCS" button. Quad View is novel in the provision of 4 display maps for the single-shot acquisition, which provide different visual representations of the arterial shear wave profile, including the elastic map (Fig. 1a), propagation map (Fig. 1b), two-dimensional reference map (Fig. 1c) and shear wave dispersion map (Fig. 1d). After recording the motion of the vascular wall for 1020 cardiac cycles, measurements were obtained from a $1.0 \mathrm{~cm}$ circular region of interest (ROI) in the systolic phase. The ROI selected in the same area as the PWV measurement. Five ROIs were placed selectively on 
the anterior and posterior walls of the bilateral carotid arteries, respectively (1 to $2 \mathrm{~mm}$ apart). Then values of SWE and SWD in electrocardiographic "R" wave were analyzed and their mean values were calculated for the final analysis.

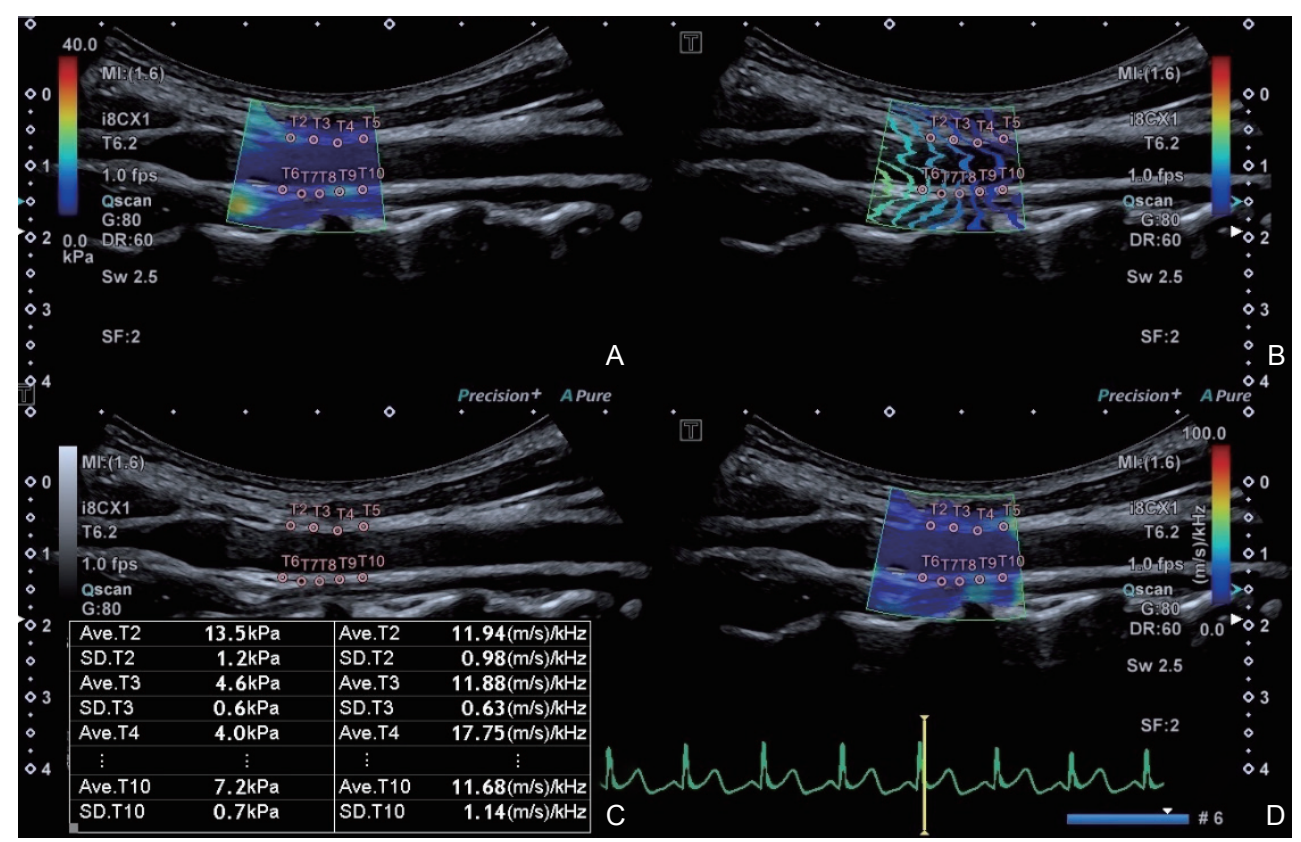

Figure 1 Quad View of shear wave profile. (A) Elastic map. The shear wave speed is coded on a color scale from blue to red, corresponding to ranging the Young modulus between 0.0 and $40.0 \mathrm{kPa}$. (B) Propagation map. The contour lines represent shear wave arrival times at different points in the tissue. (C) Two-dimensional reference map. (D) Shear wave dispersion map. The shear wave dispersion is also coded on a color scale from blue to red, corresponding to value of dispersion from 0.0 to $100.0(\mathrm{~m} / \mathrm{s}) / \mathrm{kHz}$.

\section{Statistical analysis}

Continuous data were expressed as mean \pm SD. Data were compared between two groups using twosample $t$-test for continuous data and Chi-square test or Fisher's test for categorical data. The association between the relevant factors and viscoelastic parameters were assessed with Pearson's correlation analysis. Two experienced radiologists performed the ultrasonography examinations. Repeatability evaluation between the two observers using linear correlation analysis and Bland-Altman plots. A value of $P<0.05$ was considered statistically significant. Data analysis was performed with SPSS 13.0 (SPSS, Chicago, IL, USA).

\section{Results}

\section{Demographic and clinical characteristics}

Table 1 presented the demographic and clinical characteristics of the study participants. Compared with the young group $(<50$ years old $)$, the SBP and DBP were higher in the elder group ( $\geqslant 50$ years old), while the height was shorter $(P<0.05)$. No significant differences in the biochemical parameters, gender, hypertension, and diabetes were found between the two groups $(P>0.05)$.

\section{Carotid artery structure, hemodynamics, elastic modulus and viscous index}

The ultrasound characteristics of the carotid artery were summarized in Table 2. In the elder group, CD, CIMT and PWV were higher than those of young group which indicated the arteries were remodeled in the elder subjects, increasing in radial arterial stiffness, and decreasing in arterial elasticity. On the other hand, in the young group, the SWE and SWD were all higher than the elder group which suggested that the carotid axial elasticity and viscosity were healthier. Compared with the young group, the VTI, PSV, MFV, and EDV of the carotid were significantly lower in the elder group. The two groups showed no significant difference in RI, PI, and $\mathrm{S} / \mathrm{D}$.

\section{Correlation analysis}

In all subjects, SWE was significantly negatively correlated with age, CIMT, DBP, SBP and PWV, respectively $(r=-0.291,-0.285,-0.332,-0.384$, and -0.294 , all $P<0.05)$. SWE was also significantly positively correlated with VTI, PSV, EDV and MFV, respectively $(r=0.363,0.406,0.472$, and 0.317 , all $P<0.05)$. SWD was negatively correlated with VTI, and PSV, respectively $(r=-0.492$ and -0.533 , all $P<0.05)$. 
SWD was also positively correlated with MFV $(r=$ $0.354, P<0.05)$. However, for the elder group, SWE was negatively related to SBP $(r=-0.373, P<0.05)$, and was also positively correlated with $\operatorname{EDV~}(r=0.431$, $P<0.05$ ), while SWD was negatively correlated with PWV and PSV, respectively ( $r=-0.422$ and $r=-0.372$, all $P<0.05)$. For young group, SWD positively correlated with the following factors: CD, CIMT, SBP and MFV $(r=0.531,0.462,0.377$, and 0.479 respectively, and all $P<0.05)$. SWD was negatively correlated with VTI and

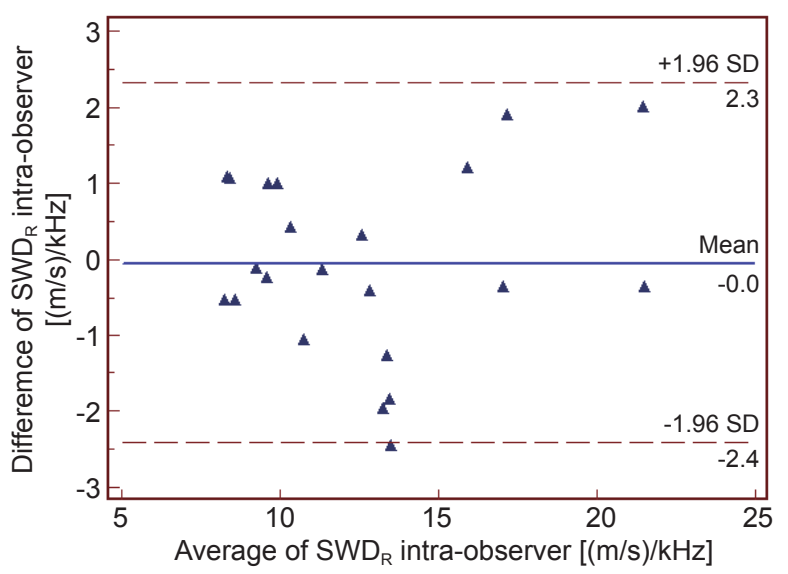

A

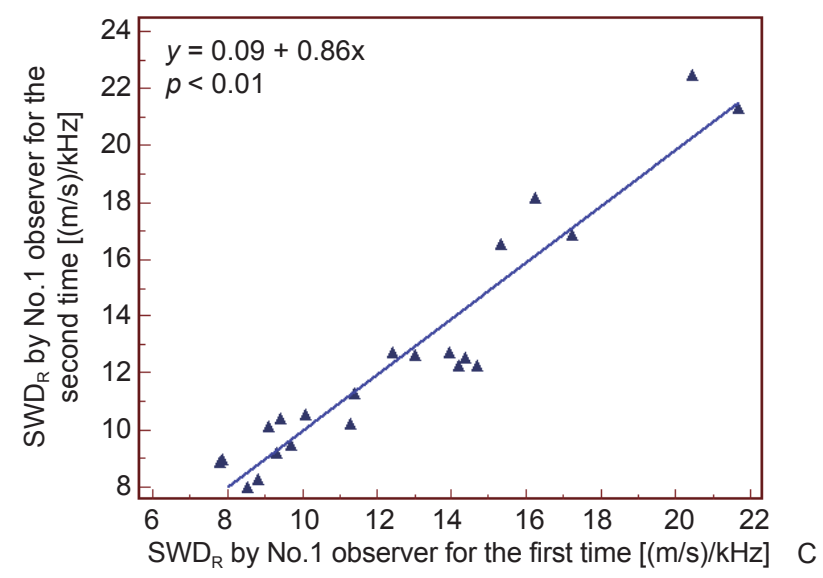

PSV respectively ( $r=-0.457$ and -0.513 , all $P<0.05)$.

\section{Inter-observer agreement}

Twenty-two subjects were randomly selected, and the SWD was repeatedly measured. The BlandAltman plots showed high repeatability of the SWD measurements obtained by one observer measuring twice (Mean difference: $-0.05+1.21(\mathrm{~m} / \mathrm{s}) / \mathrm{kHz}$ ) (Fig. 2a) and by another observer (Mean difference: $0.04+1.21(\mathrm{~m} / \mathrm{s}) /$ $\mathrm{kHz}$ ) (Fig. 2b). Results of linear correlation showed a significantly higher agreement (Fig. 2c and 2d).
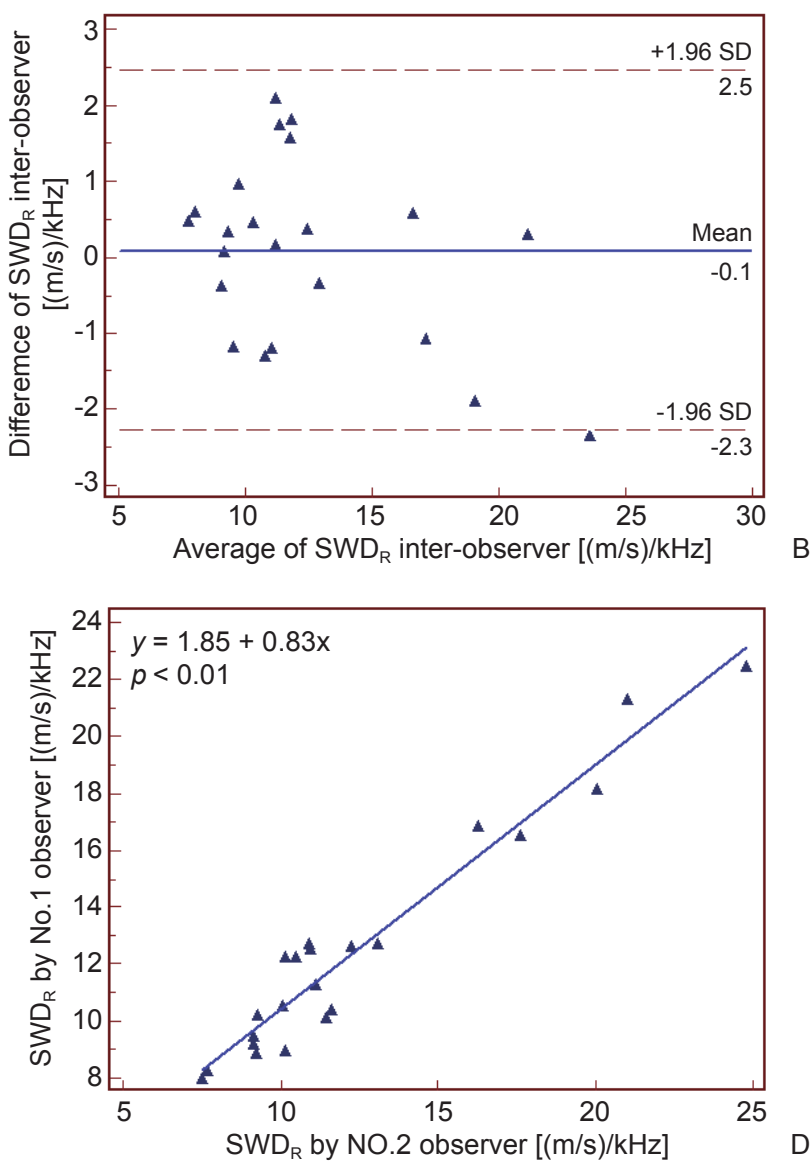

Figure 2 Repeatability test of $\mathrm{SWD}_{\mathrm{R}}$ by Bland-Altman Plots (A and B) and linear correlation analysis (C and D). Figure 2A. Repeatability test of SWD intra-observer by Bland-Altman Plots. Figure $2 \mathrm{~B}$. Repeatability test of $\mathrm{SWD}_{\mathrm{R}}$ inter-observer by Bland-Altman Plots. Figure $2 \mathrm{C}$. Repeatability test of SWD $\mathrm{R}_{\mathrm{R}}$ intra-observer by linear correlation analysis. Figure 2D. Repeatability test of $\mathrm{SWD}_{\mathrm{R}}$ inter-observer by linear correlation analysis.

\section{Discussion}

SWD imaging is an ultrasound-based technique, which can analyze shear wave propagation velocities or dispersion of the velocities at different frequencies to characterize the viscoelastic nature of tissue. Therefore, it has potential for assessment of arterial viscoelasticity. We proposed that quantitative measurements of SWD with PWV, CIMT and CD serve as the reference standard for elasticity and viscosity measurement in carotid artery. Our results showed that the values of SWD, which was an indicator of viscosity, was significantly higher in younger subjects. SWD measurement was negatively correlated with VTI, and PSV for all participants, respectively. SWD measurement was also positively correlated with MFV. Furthermore, as reported in Table 3 , the relevant factors noted differed between the two age groups. For the younger, SWD measurement correlated closely with CIMT, CD, SBP, MFV, VTI, and PSV, while for elder, SWD measurement was only significantly related with PWV and PSV. 
Table 1 Baseline and Clinical Data in healthy volunteers

\begin{tabular}{|c|c|c|c|c|}
\hline Variable & Elder group & Young group & All subjects & $P$ value \\
\hline Gender (Female/Male) & $22 / 24$ & $22 / 22$ & $44 / 46$ & 0.879 \\
\hline Age, y & $64.9 \pm 8.5$ & $32.1 \pm 8.8$ & $49.77 \pm 8.5$ & $<0.001$ \\
\hline Height, cm & $161.9 \pm 7.0$ & $166.5 \pm 6.9$ & $164.2 \pm 6.5$ & 0.024 \\
\hline Body mass index, $\mathrm{kg} / \mathrm{m}^{2}$ & $25.7 \pm 6.4$ & $22.4 \pm 4.2$ & $23.9 \pm 5.8$ & 0.133 \\
\hline Baseline SBP, mm Hg & $138.1 \pm 9.1$ & $123.6 \pm 8.2$ & $131.6 \pm 9.3$ & $<0.001$ \\
\hline Baseline DBP, mm Hg & $87.7 \pm 6.1$ & $77.6 \pm 5.9$ & $82.7 \pm 5.8$ & $<0.001$ \\
\hline History of diabetes mellitus, $n(\%)$ & $6(13.0)$ & $2(4.6)$ & $8(8.9)$ & 0.693 \\
\hline History of hypertension, $n(\%)$ & $10(21.7)$ & $6(13.6)$ & $16(17.8)$ & 0.833 \\
\hline Fasting plasma glucose, $\mathrm{m} \mathrm{mol} / \mathrm{L}$ & $6.1 \pm 1.6$ & $5.2 \pm 1.2$ & $5.7 \pm 1.4$ & 0.327 \\
\hline Total cholesterol, $\mathrm{m} \mathrm{mol} / \mathrm{L}$ & $4.5 \pm 1.1$ & $4.3 \pm 0.8$ & $4.4 \pm 0.9$ & 0.444 \\
\hline LDL cholesterol, $\mathrm{m} \mathrm{mol} / \mathrm{L}$ & $2.8 \pm 1.0$ & $2.6 \pm 0.8$ & $2.7 \pm 0.9$ & 0.421 \\
\hline Triglycerides, $\mathrm{m} \mathrm{mol} / \mathrm{L}$ & $1.6 \pm 1.2$ & $1.2 \pm 0.8$ & $1.4 \pm 1.0$ & 0.281 \\
\hline
\end{tabular}

$1 \mathrm{~mm} \mathrm{Hg}=0.133 \mathrm{kPa}$

Table 2 Characteristics of individuals with carotid ultrasound measures in two groups

\begin{tabular}{|c|c|c|c|c|}
\hline Variables & Elder group & Young group & All subjects & $P$ values \\
\hline Carotid diameter (mm) & $8.6 \pm 0.9$ & $7.0 \pm 0.5$ & $7.8 \pm 0.6$ & $<0.001$ \\
\hline Carotid intima-media thickness $(\mu \mathrm{m})$ & $687.6 \pm 138.4$ & $455.1 \pm 131.9$ & $574.9 \pm 132.2$ & $<0.001$ \\
\hline Velocity time integral (m) & $0.2 \pm 0.1$ & $0.3 \pm 0.1$ & $0.2 \pm 0.1$ & $<0.001$ \\
\hline Mean follow velocity $(\mathrm{cm} / \mathrm{s})$ & $21.4 \pm 7.1$ & $31.9 \pm 6.9$ & $26.8 \pm 6.9$ & $<0.001$ \\
\hline End-diastolic velocity $(\mathrm{cm} / \mathrm{s})$ & $13.6 \pm 7.2$ & $19.0 \pm 3.9$ & $16.2 \pm 5.2$ & 0.006 \\
\hline Velocity time integral (m) & $0.2 \pm 0.1$ & $0.3 \pm 0.1$ & $0.2 \pm 0.1$ & $<0.001$ \\
\hline Pulsatility index & $1.5 \pm 0.5$ & $1.6 \pm 0.2$ & $1.6 \pm 0.4$ & 0.311 \\
\hline Resistance index & $0.7 \pm 0.1$ & $0.7 \pm 0.1$ & $0.7 \pm 0.1$ & 0.719 \\
\hline $\mathrm{S} / \mathrm{D}$ ratio & $4.0 \pm 1.8$ & $3.9 \pm 0.8$ & $3.9 \pm 1.2$ & 0.643 \\
\hline SWE (kPa) & $10.3 \pm 8.6$ & $17.2 \pm 12.1$ & $13.8 \pm 10.8$ & 0.039 \\
\hline $\mathrm{SWD}[(\mathrm{m} / \mathrm{s}) / \mathrm{kHz}]$ & $12.1 \pm 3.5$ & $14.1 \pm 3.7$ & $13.0 \pm 3.6$ & 0.042 \\
\hline
\end{tabular}

SWD, shear wave dispersion in electrocardiographic R wave; SWE, shear wave elastography in electrocardiographic R wave

The major components in carotid wall are collagen fibers, elastic fibers and a small amount of smooth muscle cells, and these components determine the elastic and viscous properties of the artery. The relationship between stress and stain is of importance to understand the mechanism behind the CCA function [8]. The stress- strain of elasticity is expressed by the elastic modulus, which is defined as the slope of stress-strain curve. A stiffer material will have a higher elastic modulus. On the other hand, the viscosity exhibits a non-linear behavior and documents as stress-relaxation, according to the relationship between strain and time. Collagen fibers 
make a significant contribution to strength and stability of the arterial wall. They may mainly affect the viscosity of the artery and exhibit hysteresis and relaxation of strain. Elastic fibers allow the arterial wall to stretch easily [9]. $\mathrm{Xu}$ et al. respectively removed CCA from the young and elder cadaver and preformed the stress relaxation and creep tests. The results revealed that the stress relaxation and creep in the young were all significantly higher than those of elder [10]. Our study supports these study findings. Furthermore, viscous index in younger was correlated with PWV, CIMT and CD, respectively. It suggested that there might be an optimal transformation between viscosity and elasticity. It was important to note that clinical implication of measurement of arterial wall viscosity could be highly relevant to atherosclerotic plaques. When the blood pressure is stable, the creep of the arterial wall will lead to buffering through slowly increasing in vascular diameter. When vascular diameter is constant, the stress-relaxation will contribute to increasing the arterial compliance by decreasing arterial tone. In addition, this physiological process beneficial to blood supply of arterial wall. With the risk factors, the arterial stress relaxation and creep change to respond to wall remolding, corresponding to atherosclerosis.

Table 3 Correlation analysis of shear wave elasticity and viscosity parameters versus CCD risk factors

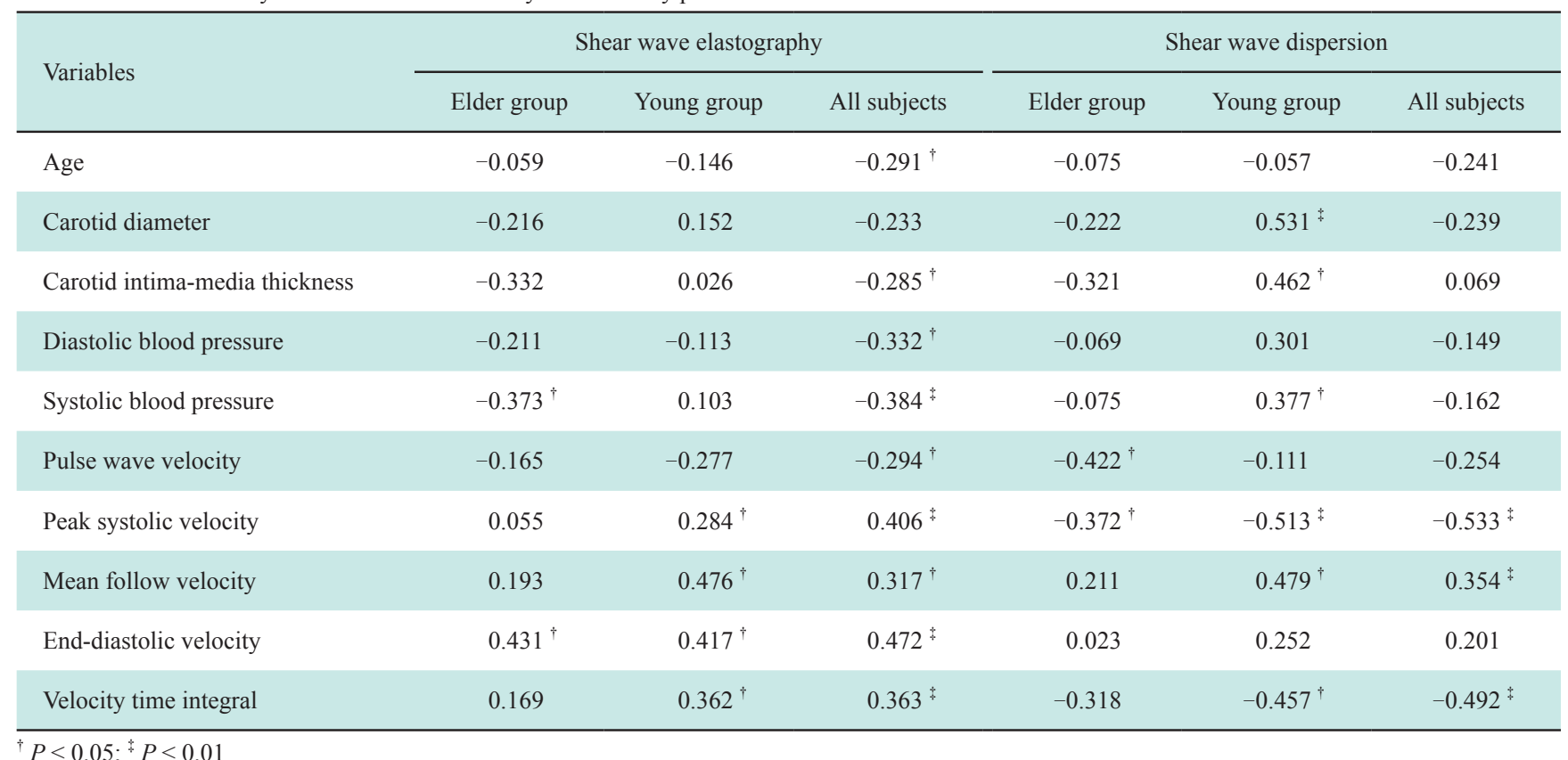

Arterial viscoelasticity is of highly non-linear and anisotropic due to their structural anisotropy [11]. The wall of an artery consists of the intima, media and adventitia. The intima is loose and is composed of endothelial cells, basement membrane, collagen fibers, elastic fibers and smooth muscle cells. The middle layer is an elastic shell, which is composed of 40-70 layers of elastic film arranged in concentric circles. The adventitia consists of loose collagen fibers and elastic fibers, which are longitudinally aligned. Three layers of arterial wall closely anchor in three dimensions and the media handles the high pressure from the heart [12]. In this study, PWV of the CCA was higher in the elder, associating with an increase in the radial elastic modulus and stiffness. Previous study had shown that the radial elasticity accounted for the arterial compliance and buffing [13]. Therefore, the end organs such as the brain were not able to accommodate the potential increase in pulsatility due to the increasing radial stiffness and decreasing compliance.

However, arterial mechanics change to respond to its wall remolding. Our study also demonstrated that the $C D$ and CIMT were increased in the elder because elastic and collagen fibers in the CCA arranged in disorder and associated with degeneration, rupture, disintegration and inflammatory as age increased [14]. It was noted that axial SWE of the elder was lower than that of the younger and negatively correlated with PWV and blood pressure, respectively. It was no agreement with the previous results [15]. It was explained as following: first, the arterial viscoelasticity was anisotropic. Though previous studies obtained the radial elastic modulus such as PWV, compliance coefficient and stiffness index, the radial elastic and longitudinal elastic modulus were confused. Second, the axial elastic modulus determined the axial deformation of the artery. Animal experiments 
had shown that the axial elastic modulus and the tension were reduced, which led to arterial distortion and remodeling.

There is a close relationship between arterial viscoelasticity and hemodynamics. Arterial stiffness can result from hemodynamic changes [16]. Blood flow acts on the vessel wall to create wall shear stress, and the vessel wall stagnates viscous blood to form a boundary layer and the particle retention. The biomechanical analysis of liquid-solid coupling has confirmed when the arterial wall is rigid (elastically infinite), the velocity of blood flow is high, the wall shear force is large, the boundary layer is thin, and the particle retention time in the boundary layer is short. Conversely, when the arterial wall is viscoelastic, the velocity of blood flow is low, the wall shear force is small, the boundary layer is thick, and the particle retention time is long. The blood flow acts on the vascular wall and leads to its deformation, so that the fluid domain of blood becomes larger, and the velocity of blood flow decreases, and vice versa. However, our study did not support these therory. We found that four hemodynamic parameters of the carotid artery in the elder group were lower than those in the young group. In general, the flow rate and flow resistance are set to be constant, and the influence of the tube wall on the fluid is observed when the liquid-solid coupling is analyzed. In the human body, the heart function of the elder may have changed [17]. In addition, changes in blood vessel shape can lead to laminar flow to be turbulent, promote particle retention, accumulate lipids and platelets, and deposit on the inner wall of the artery to occur arteriosclerosis [18]. For example, in the carotid sinus, the flow of red blood cells is biased toward the bifurcated inner wall, the oxygen dispersion of the bifurcated outer wall decreases, and the vascular endothelial cells are hypoxic, which induces an inflammatory response in the arterial wall [19].

Hemodynamics can also act on arterial stiffness. Hemodynamic factors include blood flow velocity, pressure, flow separation, secondary flow, wall shear stress, anf particle retention time, etc. Studies have shown that low-wall shear forces can lead to endothelial cell insufficiency through blood flow, induce secretion of NO and platelet-derived growth factor secretion from endothelial cells, thereby inhibiting the synthesis of vascular smooth muscle cell proteins and promoting their apoptosis, which ultimately leads to arteriosclerosis [20]. Increased wall shear force can result in intimamedia thickness increasing and leading to arteriosclerotic lesions [21]. In short, arterial viscoelasticity can affect hemodynamics, while hemodynamics counteracts the arterial wall, and forms a fluid-solid coupling, which leads to arterial disease.

There were several limitations in the current study.
Firstly, the sample size was relatively small, and the age span was large between the subgroups. Secondly, a curved-array transducer was used to evaluate the carotid viscoelasticity, while transducer of linear array could provide reliable images and measurements. Finally, the arterial viscoelasticity of the patients with cardiovascular or cerebrovascular diseases merited further investigation.

In conclusion, carotid viscoelastic properties in different age groups were presented in current study. Different behavior of carotid viscoelasticity suggested the arterial remodeling might be involved in the transformation of viscoelasticity. The clinical implication of the noninvasive measurement of arterial wall viscosity by SWD could be highly relevant to identify high-risk populations with atherosclerosis or aneurysm.

\section{Acknowledgments}

This work was supported by the Shanghai Songjiang District Science and Technology Project (18sjkjgg72 and 18sjkjgg53), Shanghai Science and Technology Committee Fund (16411969300), Shanghai Health and Family Planning Commission Fund (201640043) and Three-year Plan for Clinical Skills and Innovation in Municipal Hospitals (16CR3105B).

\section{Conflicts of Interest}

The authors declare that they have no conflict of interest.

\section{References}

[1] Maruhashi T, Soga J, Fujimura N, Idei N, Mikami S, Iwamoto $\mathrm{Y}$, et al. Endothelial dysfunction, increased arterial stiffness, and cardiovascular risk prediction in patients with coronary artery disease: FMD-j (flow-mediated dilation japan) study A. J Am Heart Assoc 2018; 7: e008588.

[2] Taniguchi R, Hosaka A, Miyahara T, Hoshina K, Okamoto H, Shigematsu K, et al. Viscoelastic deterioration of the carotid artery vascular wall is a possible predictor of coronary artery disease. $J$ Atheroscler Thromb 2015; 22: 415-423.

[3] Woodrum DA, Herrmann J, Lerman A, Romano AJ, Lerman LO, Ehman RL. Phase-contrast MRI-based elastography technique detects early hypertensive changes in ex vivo porcine aortic wall. $J$ Magn Reson Imaging 2009; 29: 583-587.

[4] Maksuti E, Widman E, Larsson D, Urban MW, Larsson M, Bjällmark A. Arterial stiffness estimation by shear wave elastography: validation in phantoms with mechanical testing. Ultrasound Med Biol 2016; 42: 308-321.

[5] Couade M, Pernot M, Prada C, Messas E, Emmerich J, Bruneval P, et al. Quantitative assessment of arterial wall biomechanical properties using shear wave imaging. Ultrasound Med Biol 2010; 36: 1662-1676.

[6] Maksuti E, Bini F, Fiorentini S, Blasi G, Urban MW, Marinozzi F, et al. Influence of wall thickness and diameter on arterial shear wave elastography: a phantom and finite element study. Phys Med Biol 2017; 62: 2694-2718.

[7] Li Z, Du L, Wang F, Luo X. Assessment of the arterial stiffness in patients with acute ischemic stroke using longitudinal elasticity modulus measurements obtained with shear wave elastography. Med Ultrason 2016; 18: 182-189.

[8] Bell JS, Adio AO, Pitt A, Hayman L, Thorn CE, Shore AC, et al. 
Microstructure and mechanics of human resistance arteries. Am $J$ Physiol Heart Circ Physiol 2016; 311: H1560-H1568.

[9] Osidak MS, Osidak EO, Akhmanova MA, Domogatsky SP, Domogatskaya AS. Fibrillar, fibril-associated and basement membrane collagens of the arterial wall: architecture, elasticity and remodeling under stress. Curr Pharm Des 2015; 21: 1124-1133.

[10] Scuteri A, Chen CH, Yin FC, Chih-Tai T, Spurgeon HA, Lakatta EG. Functional correlates of central arterial geometric phenotypes. Hypertension 2001; 38: 1471-1475.

[11] Li D, Xu D, Li P, Wei J, Yang K, Zhao C. Viscoelastic evaluation of fetal umbilical vein for reconstruction of middle cerebral artery. Neural Regen Res 2013; 8: 3055-3062.

[12] Charalambous HP, Roussis PC, Giannakopoulos AE. Viscoelastic dynamic arterial response. Comput Biol Med 2017; 89: 337-354.

[13] Li Z, Du L, Wang F, Luo X. Assessment of the arterial stiffness in patients with acute ischemic stroke using longitudinal elasticity modulus measurements obtained with shear wave elastography. Med Ultrason 2016; 18: 182-189.

[14] Roy Choudhury K, Skwerer S. Branch order regression for modeling brain vasculature. Med Phys 2018; 45: 1123-1134.
[15] Luo XH, Zhang JH, Shao SH, Yan MH, Li ZH. The role of ultrasound shear wave dispersion imaging in evaluating carotid viscoelasticity: a preliminary study. Advanced Ultrasound in Diagnosis and Therapy 2019; 3: 97-102.

[16] Hashimoto J. Central hemodynamics for management of arteriosclerotic diseases. $J$ Atheroscler Thromb 2017; 24: 765-778.

[17] Keller KM, Howlett SE. Sex differences in the biology and pathology of the aging heart. Can J Cardiol 2016; 32: 1065-1073.

[18] Petrini J, Ring M, Franco-Cereceda A, Caidahl K, Eriksson MJ. Aortic versus carotid intima-media thickness and impact of aortic valve disease. Clin Physiol Funct Imaging 2018; 38: 895-902.

[19] Ferns GAA, Heikal L. Hypoxia in atherogenesis. Angiology 2017; 68:472-493.

[20] Mohamied Y, Sherwin SJ, Weinberg PD. Understanding the fluid mechanics behind transverse wall shear stress. J Biomech 2017; 50: 102-109.

[21] Kang C. Using ultrasound radio frequency technology to assess regression of the structure and function of the carotid artery by radioiodine therapy in hyperthyroidism patients. Arch Med Sci 2015; 11: 1236-1243. 